\title{
Constraining the Higgs boson mass: a non-perturbative lattice study
}

\author{
Karl Jansen* \\ NIC, DESY, Platanenallee 6, D-15738 Zeuthen, Germany \\ E-mail: karl.jansen@desy.de \\ John Bulava \\ CERN, Physics Department, CH-1211 Geneva 23, Switzerland \\ E-mail: john.bulava@cern.ch
}

\section{Attila Nagy}

NIC, DESY, Platanenallee 6, D-15738 Zeuthen, Germany

and

Humboldt-Universität zu Berlin, Institut für Physik, Newtonstr. 15, D-12489 Berlin, Germany

E-mail: attila.nagy@physik.hu-berlin.de

We present non-perturbatively obtained results for upper and lower Higgs boson mass bounds using a chiral invariant lattice formulation of the Higgs-Yukawa sector of the standard model. We determine the mass bounds both, for a standard model top quark mass and for a possible fourth quark generation with masses up to $700 \mathrm{GeV}$.

Xth Quark Confinement and the Hadron Spectrum,

October 8-12, 2012

TUM Campus Garching, Munich, Germany

${ }^{*}$ Speaker. 


\section{Introduction}

The discovery of a scalar particle at the Large Hadron Collider (LHC) [1, 2] bears many interesting consequences. If this scalar particle turns out not to be the standard model Higgs boson, there would be a first indication about the physics beyond the standard model. If, on the other hand, it will turn out to be the standard model Higgs boson, we have the possibility to determine the energy scale up to which the standard model can be maximally valid.

The reason is that, due to triviality, in the standard model the cut-off cannot be removed. However, theoretically, lower and upper bounds on the Higgs boson mass as a function of the cut-off can be given. Therefore, once the Higgs boson mass is determined, it becomes possible to compute the value of the cut-off where the Higgs boson mass becomes incompatible with the theoretical mass bounds. This, in turn, will then provide the largest possible energy scale where the standard model has to be replaced by some new, yet unknown physics.

In the light of a Higgs boson mass of $125-126 \mathrm{GeV}$, it is in particular the lower Higgs boson mass bound that can provide the values of the cut-off and hence the energy scale where new physics beyond the standard model has to appear. In fact, within perturbation theory the SM could be valid up to very high energies before violating the Higgs boson mass bounds, see ref. [3] for a recent analysis at next-to-next leading order of perturbation theory.

When looking at the Higgs-Yukawa sector of the standard model, which is most relevant for the Higgs boson mass bounds, in principle, the involved couplings of the theory, the quartic selfinteraction of the Higgs field and the Yukawa-coupling between the Higgs field and the fermions, can grow strong. This happens, when the involved masses are large and then perturbation theory might fail to analyze the theory. The Higgs boson mass bounds are indeed examples where the applicability of perturbation theory is questionable. The first is the upper Higgs boson mass bound which is based on triviality arguments [4]. Here the Higgs boson mass can become large, resulting in a strong value of the quartic coupling such that perturbation theory may not work anymore. The second is the lower Higgs boson mass bound which is based on vacuum instability arguments [5, 6, $7,8]$. Here it is unclear whether this instability is not an artefact of perturbation theory applied at large values of the Higgs field such that an expansion around the minimum of the effective potential is not justified anymore. Another example where non-perturbative calculations are necessary is the possibility of a heavy fourth fermion generation $[9,10]$ which would lead to a large value of the corresponding Yukawa coupling.

Therefore, it would be very desirable to have ab-initio, non-perturbative computations of the Higgs boson mass bounds for which lattice field theory techniques would offer a computational strategy to address possible non-perturbative effects. However, for many years such calculations were blocked by the inability to implement a chiral symmetric Higgs-Yukawa interaction on a lattice, as demanded by the continuum theory.

Conceptually clean investigations of Higgs-Yukawa models on the lattice became possible when it was realised that -based on the Ginsparg-Wilson relation [11]- there exists a consistent formulation of an exact lattice chiral symmetry [12], which emulates the chiral character of the Higgs-fermion coupling structure of the SM. This triggered a number of lattice investigations of Higgs-Yukawa like models [13, 14, 15, 16, 17, 18, 19, 20, 21].

A systematic investigation of the phase structure of the theory and Higgs boson mass bounds, 
including a study of the Higgs boson as a resonance, were carried through in refs. [16, 17, 19, 21, 22, 23], see also ref. [24] for a recent review. In this proceedings contribution, we will report about the status of the calculations for the lower and upper Higgs boson mass bounds for fermion masses covering the standard model top quark mass up to quark masses of about $700 \mathrm{GeV}$.

\section{The lattice model}

One important ingredient of the Standard Model is the chiral structure of the scalar-fermion interactions which should be kept on a 4-dimensional Euclidean space-time lattice. This has been a long-standing obstacle to the lattice regularization of Higgs-Yukawa models and was finally overcome by employing the Neuberger 'Overlap' $[12,25,26]$ discretization of the fermion action.

Following the proposition in ref. [12] it is therefore possible to construct a lattice HiggsYukawa model with a global $S U(2)_{L} \times U(1)_{Y}$ symmetry. Specifically, the fields included in our model are a scalar doublet $\varphi$ and two fermions, the left-handed components of which are paired into an $S U(2)$ doublet. The lattice action can thus be written as

$$
\begin{gathered}
S=S_{F}+S_{\phi}, \quad S_{F}=\sum_{x y} \bar{\psi}_{x} M_{x y} \psi_{y}, \\
S_{\phi}=-\kappa \sum_{x, \mu} \operatorname{Tr} \phi_{x}^{\dagger}\left[\phi_{x+\mu}+\phi_{x-\mu}\right]+\sum_{x} \operatorname{Tr} \phi_{x}^{\dagger} \phi_{x} \\
+\hat{\lambda} \sum_{x} \operatorname{Tr}\left(\phi_{x}^{\dagger} \phi_{x}-1\right)^{2}, \\
M_{x y}=D_{x y}^{o v} 1_{2 \times 2}+y\left(P_{+} \phi_{x}^{\dagger} \hat{P}_{+}+P_{-} \phi_{x} \hat{P}_{-}\right) \delta_{x y},
\end{gathered}
$$

where $\psi$ is a doublet of four-component spinor fields, $D^{o v}$ is the free Overlap Dirac operator with a Wilson kernel and $\phi_{x}$ denotes the scalar field represented by a complex $2 \otimes 2$ matrix. The left- and right-handed projection operators $P_{ \pm}$and the modified projectors $\hat{P}_{ \pm}$are given by

$$
\begin{gathered}
P_{ \pm}=\frac{1 \pm \gamma_{5}}{2}, \quad \hat{P}_{ \pm}=\frac{1 \pm \hat{\gamma}_{5}}{2}, \\
\hat{\gamma}_{5}=\gamma_{5}\left(1-\frac{1}{\rho} D^{o v}\right) .
\end{gathered}
$$

The action introduced above obeys an exact global $\mathrm{SU}(2)_{L} \times U(1)_{Y}$ lattice chiral symmetry. For $\Omega_{L} \in \mathrm{SU}(2)$ and $\theta \in[0,2 \pi]$ the action is invariant under the transformation

$$
\begin{aligned}
\psi & \rightarrow U_{Y} \hat{P}_{+} \psi+U_{Y} \Omega_{L} \hat{P}_{-} \psi, \\
\bar{\psi} & \rightarrow \bar{\psi} P_{+} \Omega_{L}^{\dagger} U_{Y}^{\dagger}+\bar{\psi} P_{-} U_{Y}^{\dagger}, \\
\phi & \rightarrow U_{Y} \phi \Omega_{L}^{\dagger}, \phi^{\dagger} \rightarrow \Omega_{L} \phi^{\dagger} U_{Y}^{\dagger}
\end{aligned}
$$

with $U_{Y} \equiv \exp (i \theta Y)$, where $Y$ labels the representation of the global hypercharge symmetry group $U(1)_{Y}$. It should be noted that in the continuum limit the (global) continuum $\mathrm{SU}(2)_{L} \times \mathrm{U}(1)_{Y}$ chiral symmetry is recovered.

We can relate the parameters and fields appearing in Eq. 2.1 to those appearing in the standard scalar complex doublet continuum Lagrangian $\left(\mathscr{L}=\left|\partial_{\mu} \varphi\right|^{2}+\frac{1}{2} m_{0}^{2}|\varphi|^{2}+\lambda|\varphi|^{4}\right)$ by

$$
\begin{gathered}
\varphi_{x}=\sqrt{2 \kappa}\left(\begin{array}{c}
\phi_{x}^{2}+i \phi_{x}^{1} \\
\phi_{x}^{0}-i \phi_{x}^{3}
\end{array}\right), \\
\lambda=\frac{\hat{\lambda}}{4 \kappa^{2}}, \quad m_{0}^{2}=\frac{1-2 \hat{\lambda}-8 \kappa}{\kappa} .
\end{gathered}
$$




\subsection{Observables}

The cutoff in our model is provided by the inverse lattice spacing $(\Lambda=1 / a)$ and we set the physical value of $\Lambda$ using the phenomenological Higgs field vacuum expectation value $\left(v_{R}\right)$,

$$
\left(\sqrt{2} G_{F}\right)^{-\frac{1}{2}} \sim 246 \mathrm{GeV}=\frac{v_{R}}{a} \equiv \frac{v}{\sqrt{Z_{G}} \cdot a},
$$

where $Z_{G}$ denotes the Goldstone boson field renormalization constant and $v$ the bare scalar field vacuum expectation value. We target a mass range for the degenerate fermion doublet of $175 \mathrm{GeV} \lesssim$ $m_{f} \lesssim 700 \mathrm{GeV}$, while fixing the cutoff at $\Lambda \approx 1.5 \mathrm{TeV}$. Although the masses of the fermion doublet are degenerate in this work, we plan to assess the effect of a mass splitting in the near future. At $m_{f}=m_{t}=175 \mathrm{GeV}$ the splitting $m_{b}-m_{t}$ has been taken into account and found to have a small effect on the lower Higgs boson mass bound which, moreover, can be taken into account by the effective potential evaluated in lattice perturbation theory [19].

Details on the simulation algorithm, which is based on the Hybrid Monte Carlo algorithm [27] and its extension of ref. [28], can be found in ref. [20].

It has been demonstrated [19] that the Higgs boson mass is a monotonically increasing function of the bare quartic coupling $\hat{\lambda}$. Therefore, the lower bound for the Higgs boson mass at fixed cutoff and $m_{f}$ is obtained at $\hat{\lambda}=0$, while the upper bound is obtained at $\hat{\lambda}=\infty$ [21].

Since we work in a finite volume with no external symmetry breaking source, the naively defined vacuum expectation value is zero in an ensemble average. We therefore follow the strategy described and tested in refs. [29, 30,31], of rotating a given scalar field configuration to a preferred direction. It can be shown that in infinite volume this leads to the same vacuum expectation value as obtained in the standard procedure involving the limit of vanishing external source [31].

The determination of the Goldstone boson renormalization constant $Z_{G}$, the Higgs boson mass $m_{H}$, and the fermion mass $m_{f}$ have been discussed in detail in ref. [21] and are briefly reviewed here. The renormalization constant $Z_{G}$ is computed from the slope of the inverse Goldstone boson propagator at vanishing Euclidean four-momentum transfer, in the standard on-shell scheme. Operationally, this constant is obtained from fits to the propagator at small momenta.

Due to the existence of massless Goldstone modes in the spontaneously broken phase of our theory, the finite size effects are not exponential as it is the case in theories with a mass gap, but algebraic. In particular, the finite size effects vanish with inverse even powers of the spatial extent of the lattice. This is illustrated in fig. 1 at the examples of the renormalized scalar field expectation value and the Higgs boson mass. The figure also shows that the finite size effects can be substantial and that large lattices are required to perform a reliable infinite volume extrapolation which has indeed been performed for all results discussed, if not indicated otherwise.

Another effect of the massless Goldstone modes is that the Higgs boson is unstable and can decay to final states containing an even number of Goldstone bosons. We employ two definitions of the Higgs boson mass which both ignore this finite decay width. The Higgs boson propagator mass $m_{H}^{P}$ is derived from fits to the momentum space Higgs boson propagator defined as

$$
\tilde{G}_{H}(p)=\left\langle\tilde{h}_{p} \tilde{h}_{-p}\right\rangle, \tilde{h}_{p}=\frac{1}{\sqrt{L_{s}^{3} \cdot L_{t}}} \sum_{x} e^{-i p x} h_{x}
$$

while the correlator mass $m_{H}^{C}$ is derived from fits to the Higgs boson temporal correlation function. Although finite decay width corrections to these formulae are quite different, the mass extracted 


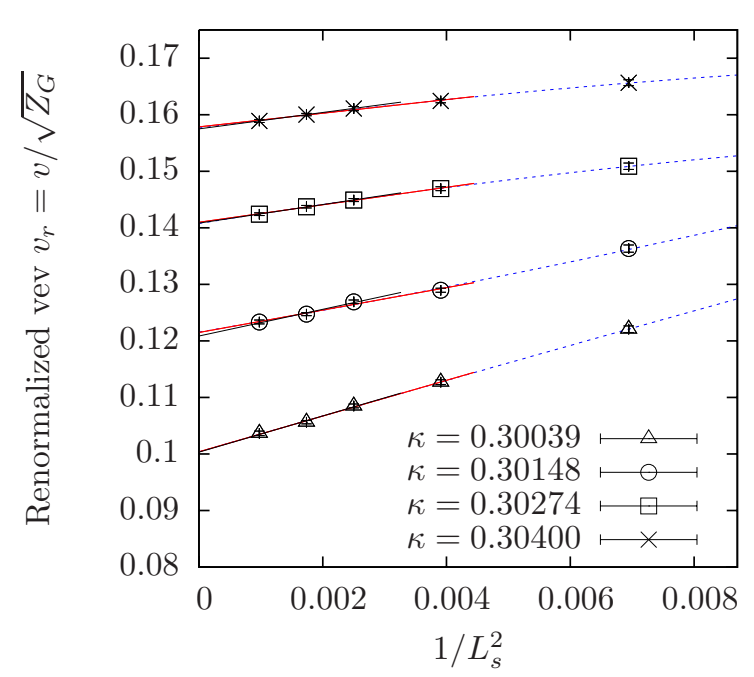

(a) Dependence of the renormalized vev $v_{r}=v / \sqrt{Z_{G}}$ on the squared inverse lattice side length $1 / L_{s}^{2}$ at infinite bare quartic coupling constant.

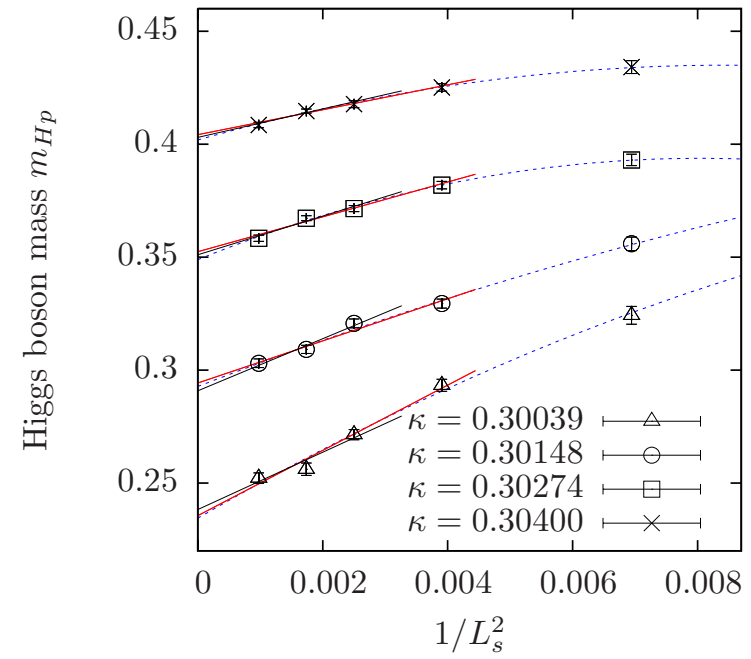

(b) Dependence of the Higgs boson propagator mass $m_{H p}$ on the squared inverse lattice side length $1 / L_{S}^{2}$ at infinite bare quartic coupling constant.

Figure 1: The dependence of the renormalized vev $v_{r}=v / \sqrt{Z_{G}}$ and the Higgs boson propagator mass $m_{H p}$ on the squared inverse lattice side length $1 / L_{s}^{2}$ is presented. In all plots the dashed curves display a quadratic fit in $1 / L_{s}^{2}$ with lower threshold values $L_{s}^{\prime}=16$, while the solid lines depict the linear fits in $1 / L_{s}^{2}$ with lower threshold values $L_{s}^{\prime}=20$.

from both of these procedures typically differs by less than $10 \%$, lending credence to our approximation of a stable Higgs boson. Furthermore, a rigorous study of the Higgs boson decay width at non-vanishing external source has been performed at $m_{f}=m_{t} \sim 175 \mathrm{GeV}$ in ref. [23] which obtained a narrow decay width for all values of the bare quartic coupling, further supporting the validity of the stable Higgs boson approximation. For this work we quote $m_{H}^{P}$ as the central value as it is typically the lowest estimate of $m_{H}$.

Finally, we compute the quark mass from the exponential decay of the temporal correlation function $C_{f}(t)$ at large Euclidean time separations $t$, defined as

$$
C_{f}(t)=\frac{1}{L_{s}^{6}} \sum_{\vec{x}, \vec{y}} \operatorname{Re} \operatorname{Tr}\left(f_{L, 0, \vec{x}} \cdot \bar{f}_{R, t, \vec{y}}\right),
$$

where the left- and right-handed spinors are defined using the projection operators in Eq. 2.2.

As an example, we show here the results of the Higgs boson mass bound calculations where the upper and lower bounds were computed at several choices of the cut-off scale and fermion masses $m_{f}$. The main result is shown in fig. 2, where in the left graph, the situation for a SM top quark mass is shown. The right graph shows, at a fixed value of the cut-off of $1.5 \mathrm{TeV}$, the mass dependence of the lower and upper Higgs boson mass bounds as a function of the fermion mass, see also [22]. It can be clearly seen that while the upper bound is relatively unaffected when using a heavy fermion mass, the lower bound increases substantially.

The graphs in fig. 2 have a very interesting interpretation concerning the validity of the SM and the existence of a fourth fermion generation. For the SM, a Higgs boson mass of about $125 \mathrm{GeV}$ just seems to escape the Higgs boson mass bounds leading the SM to be valid up to very high 


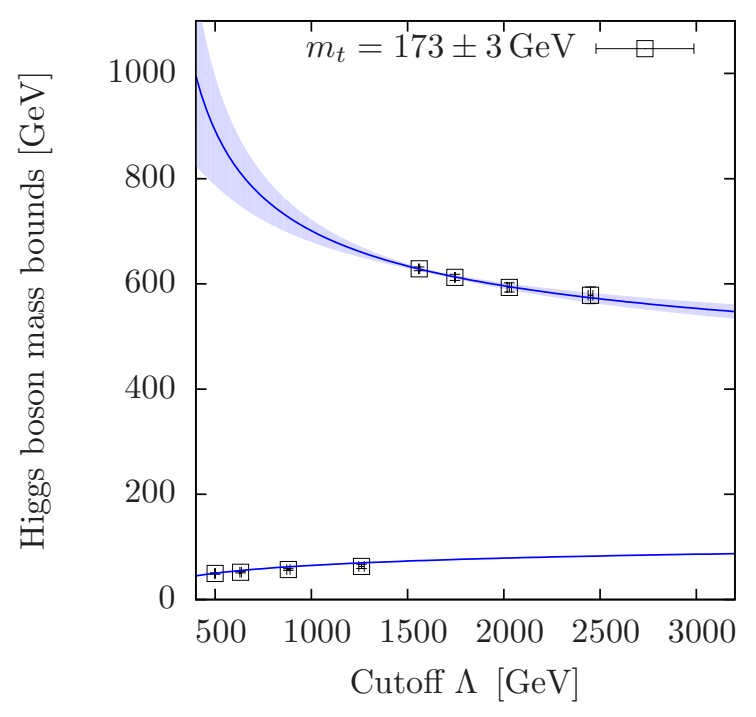

(a) The cut-off dependence of the upper and lower Higgs boson mass bounds for a fermion mass at $\sim 173 \mathrm{GeV}$.

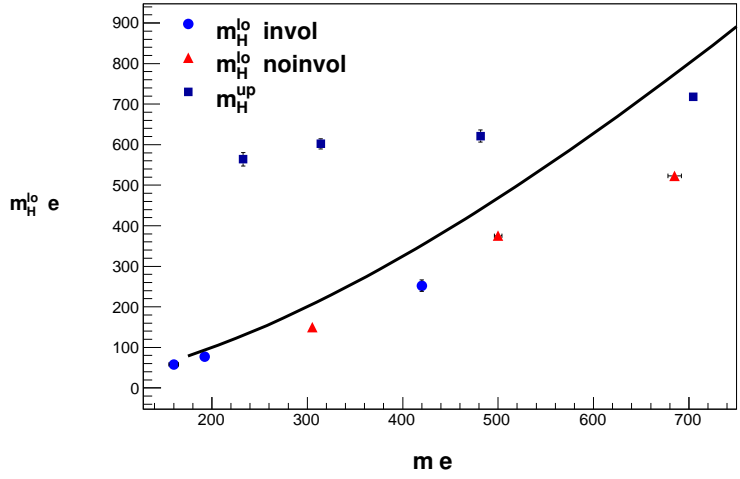

(b) The mass dependence of the lower and upper Higgs boson mass bounds at a cut-off of about $1.5 \mathrm{TeV}$. The solid line represents a perturbative lattice effective potential calculation for the lower Higgs boson mass bound.

Figure 2: The cut-off and fermion mass dependence of the lower and upper Higgs boson mass bounds.

energies. On the other hand, in this scenario, a fourth fermion generation seems to be ruled out for fermion masses larger than about $300 \mathrm{GeV}$. Combining this with phenomenological analyses of allowed fermion masses to be larger than about $500 \mathrm{GeV}$, this indicates that a straightforward extension of the SM with a fourth fermion generation is not compatible with the experimental finding of a possible $125 \mathrm{GeV}$ Higgs boson mass.

As mentioned above, for the Higgs-Yukawa model considered here, the Higgs boson has also been treated as a true resonance [23]. It was found that the Higgs boson resonance width does not exceed about $10 \%$ of the resonance mass for all values of the quartic coupling considered. Thus, the Higgs boson can be considered as a narrow resonance and the mass bounds shown in fig. 2, which were obtained assuming a stable Higgs boson, are not affected by resonance effects.

\section{Concluding remarks}

In this proceedings contribution we have shown that lattice field theory can provide lower and upper Higgs boson mass bounds. This is a remarkable result since for many years lattice computations within Higgs-Yukawa models were blocked by the difficulty to implement the continuum chiral symmetric nature of the Higgs-fermion interaction. The situation only changed when, through the Ginsparg-Wilson relation, a consistent formulation of a Higgs-Yukawa theory could be given.

Within this framework, we have here given bounds for the Higgs boson mass, see fig. 2. We have found that the upper bound is only a mildly increasing function with increasing fermions. On the other hand, the lower bound changes substantially when the fermion mass is increased. This led then to the conclusion that a straightforward fourth fermion generation is excluded. 
An open question is, whether the lower Higgs boson mass bound can be altered by adding higher dimensional operators. The key to answer this question is an analysis of the effective potential, evaluated in lattice perturbation theory using the same lattice formulation as employed in the numerical simulations. It has been found that for a standard model top quark mass such a perturbative calculation reproduces the simulation results very precisely [19]. When the fermion mass is increased above the top quark mass, such an effective potential calculation still describes the simulation results for the lower Higgs boson mass bound rather well, although not with the same precision as for the case of the top quark mass, as can be seen in fig. 2(b) where the outcome of the lattice effective potential calculation is represented with the solid line, see also ref. [24].

As a consequence, the lattice perturbative effective potential calculation can be used to study the effect of a higher dimensional operator. Taken as an example a $\lambda_{6} \phi^{6}$ term, a variation of $\lambda_{6}$ in the range $\lambda_{6}=[0.0,0.1]$ results in a less than $15 \%$ difference in the lower bound. Thus, the lower Higgs boson mass bound is rather unaffected by such higher dimensional operator. This stability of the lower bound leaves then very little room for the existence of a straightforward fourth fermion generation. Of course, this result from perturbation theory should be scrutinized by direct, nonperturbative numerical simulations, a task we want to perform in the future.

\section{Acknowledgments}

We thank George Hou, Bastian Knippschild, David Lin, Kei-ichi Nagai, and Kenji Ogawa for a very fruitful collaboration. We also thank M. Müller-Preussker for his continuous support and him as well as Ulli Wolff for helpful discussions. Many of the results presented here are based on the work of Philipp Gerhold and Jim Kallarackal whom we thank at this point to have built the basis for our calculations and to let us freely use their simulation and analysis programmes. We moreover acknowledge the support of the DFG through the DFG-project Mu932/4-2. The numerical computations have been performed on the HP XC4000 System at the Scientific Supercomputing Center Karlsruhe and on the SGI system HLRN-II at the HLRN Supercomputing Service Berlin-Hannover.

\section{References}

[1] The ATLAS Collaboration, G. Aad et al., 1207.7214.

[2] The CMS Collaboration, S. Chatrchyan et al., 1207.7235.

[3] G. Degrassi et al., 1205.6497.

[4] R. F. Dashen and H. Neuberger, Phys.Rev.Lett. 50, 1897 (1983).

[5] T. Lee and G. Wick, Phys.Rev. D9, 2291 (1974).

[6] A. D. Linde, JETP Lett. 23, 64 (1976).

[7] S. Weinberg, Phys.Rev.Lett. 36, 294 (1976).

[8] M. Sher, Phys.Rept. 179, 273 (1989).

[9] M. S. Carena, A. Megevand, M. Quiros and C. E. Wagner, Nucl.Phys. B716, 319 (2005), [hep-ph/0410352].

[10] B. Holdom et al., PMC Phys. A3, 4 (2009), [0904.4698]. 
[11] P. H. Ginsparg and K. G. Wilson, Phys.Rev. D25, 2649 (1982).

[12] M. Luscher, Phys.Lett. B428, 342 (1998), [hep-lat/9802011].

[13] T. Bhattacharya, M. R. Martin and E. Poppitz, Phys.Rev. D74, 085028 (2006), [hep-lat/0605003].

[14] J. Giedt and E. Poppitz, JHEP 0710, 076 (2007), [hep-lat/0701004].

[15] E. Poppitz and Y. Shang, JHEP 0708, 081 (2007), [0706.1043].

[16] P. Gerhold and K. Jansen, JHEP 0709, 041 (2007), [0705.2539].

[17] P. Gerhold and K. Jansen, JHEP 0710, 001 (2007), [0707.3849].

[18] Z. Fodor, K. Holland, J. Kuti, D. Nogradi and C. Schroeder, PoS LAT2007, 056 (2007), [0710.3151].

[19] P. Gerhold and K. Jansen, JHEP 0907, 025 (2009), [0902.4135].

[20] P. Gerhold, 1002.2569.

[21] P. Gerhold and K. Jansen, JHEP 1004, 094 (2010), [1002.4336].

[22] P. Gerhold, K. Jansen and J. Kallarackal, JHEP 1101, 143 (2011), [1011.1648].

[23] P. Gerhold, K. Jansen and J. Kallarackal, Phys.Lett. B710, 697 (2012), [1111.4789].

[24] J. Bulava et al., 1210.1798.

[25] H. Neuberger, Phys.Lett. B417, 141 (1998), [hep-lat/9707022].

[26] H. Neuberger, Phys.Lett. B427, 353 (1998), [hep-lat/9801031].

[27] S. Duane, A. D. Kennedy, B. J. Pendleton and D. Roweth, Phys. Lett. B195, 216 (1987).

[28] R. Frezzotti and K. Jansen, Phys.Lett. B402, 328 (1997), [hep-lat/9702016].

[29] A. Hasenfratz et al., Z.Phys. C46, 257 (1990).

[30] A. Hasenfratz et al., Nucl.Phys. B356, 332 (1991).

[31] M. Gockeler and H. Leutwyler, Nucl.Phys. B361, 392 (1991). 